\title{
Predicting the onset of metal-insulator transitions in transition metal oxides-a first step in designing neuromorphic devices
}

\author{
Shenli Zhang ${ }^{1}$, Hien Vo² and Giulia Galli1,2,3 \\ ${ }^{1}$ Pritzker School of Molecular Engineering, The University of Chicago, Chicago, IL, USA; \\ ${ }^{2}$ Department of Chemistry, The University of Chicago, Chicago, IL, USA; ${ }^{3}$ Argonne National \\ Laboratory, Lemont, IL, USA
}

1. Calculations of the dielectric constant

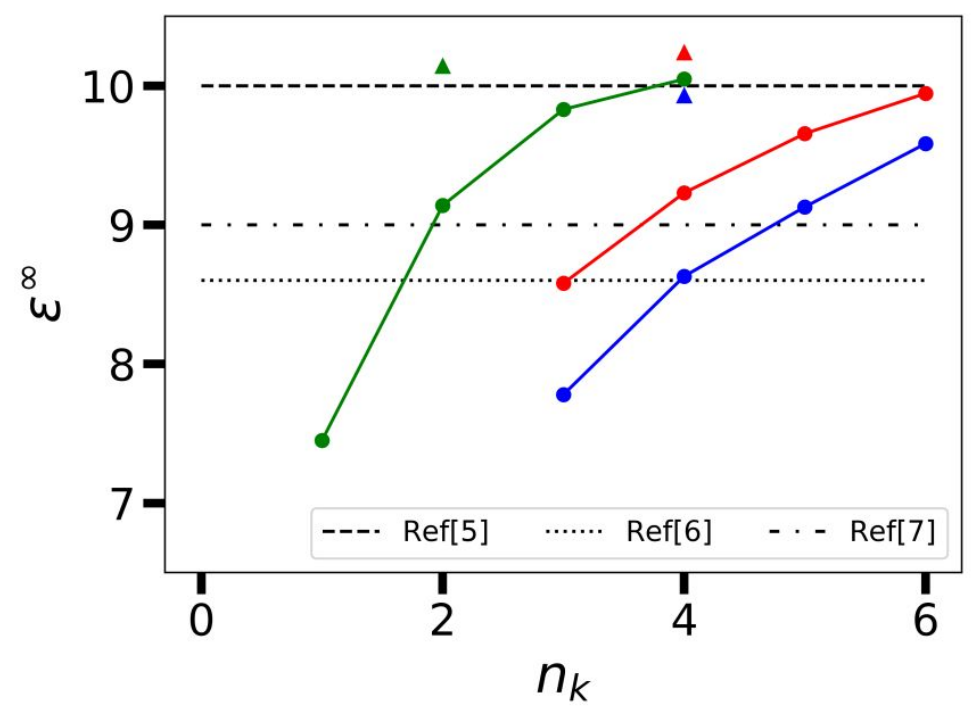

Figure S1 The componenet of the dielectric constant $\varepsilon^{\infty}$ of the pristine LCO phase computed along the three axes, a (red), b (green) and c (blue), of the orthorhombic cell (see Fig. 1 in the main manuscript), as a function of the linear number of k-points $n_{k}$ included in the calculation (the total number of point is $n_{k}^{3}$ ). We show results obtained with density functional perturbation theory (DFPT $+U)^{1,2}$ calculations (triangles) and from Berry phase $\mathrm{e}^{3,4}$ calculations (circles). Results from Berry phase calculations are consistent with those of DFPT, although a finer k-point mesh is required to obtain convergence. Ref[5] and Ref[6] both reports experimental values for single crystals measured at room temperature ; Ref[7] reports DFT $+U$ calculations with a $U$ larger $(U=5$ $\mathrm{eV}$ ) than used in our calculations $(U=3 \mathrm{eV})$, which may be responsible for the differences in values found here and in Ref.7 (1\%). 


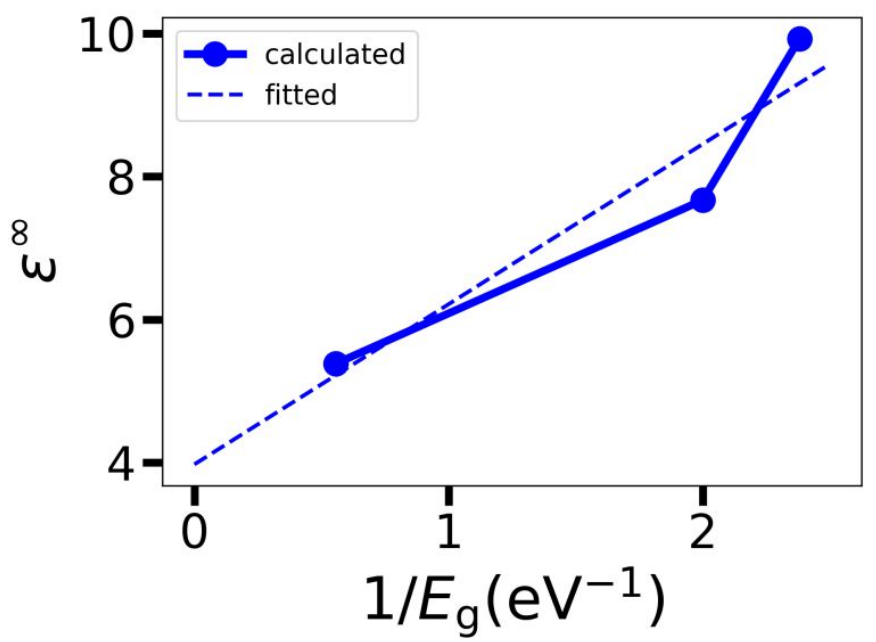

Figure S2 Linear fit of computed values of $\varepsilon^{\infty}$ as a function of the inverse of the band gap. The three points are computed for the perovskite structure (0\% vacancies), $8.3 \%$ vacancy concentration and the BM (16.7\%) structure.

Table S1 Calculated values of the dielectric constant $\varepsilon^{\infty}$ along three axes for three LCO perovskite structures, corresponding to $0 \%, 8.3 \%$ and $16.7 \%$ oxygen vacancy concentration, using DFPT $+U$. The value for $12.5 \%$ vacancy concentration was obtained from the linear fit shown in Fig. S2. The axes $a, b$ and $c$ are defined in Fig. 1 in the main manuscript.

\begin{tabular}{|l|l|l|l|l|}
\hline Axis & $\begin{array}{l}\text { Perovskite } \\
(0 \%)\end{array}$ & $\begin{array}{l}2 \text { vacancies } \\
(8.3 \%)\end{array}$ & $\begin{array}{c}3 \text { vacancies } \\
(12.5 \%)\end{array}$ & \multicolumn{1}{c|}{$\begin{array}{c}\text { BM } \\
(16.7 \%)\end{array}$} \\
\hline a & 10.24 & 7.67 & 6.46 & 5.37 \\
\hline c & 9.93 & 7.67 & & 5.38 \\
\cline { 1 - 2 } b & 10.14 & 7.37 & & 5.35 \\
\hline
\end{tabular}

2. Calculations of the permanent polarization

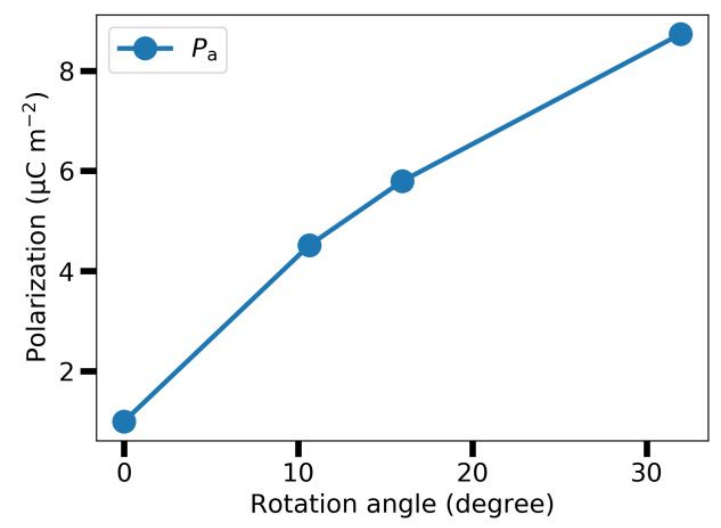

Figure S3 Computed polarization along the a axis (see Fig. 1 in the main manuscript) as a function of the tetrahedral rotation angle in the two-vacancy structure. 


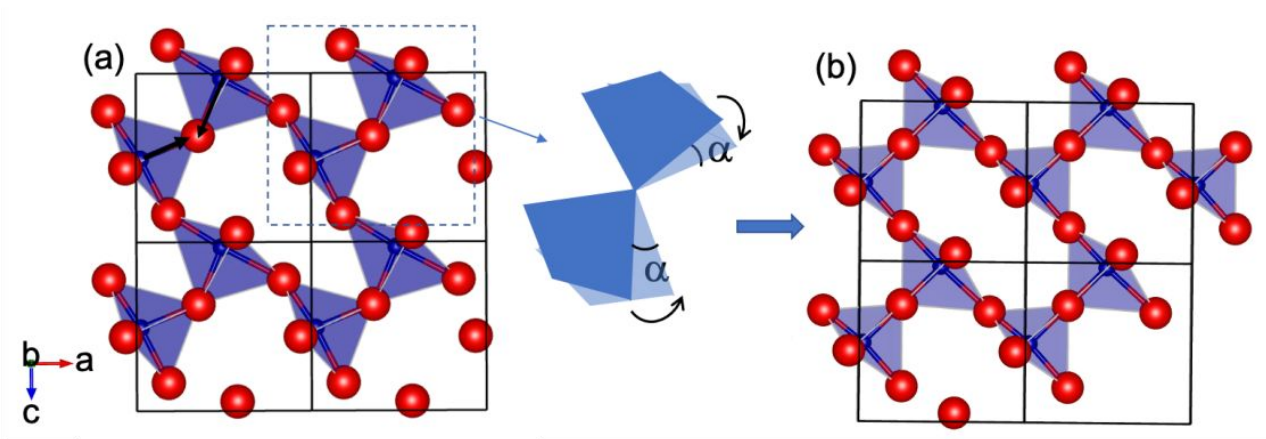

Figure S4 Polarization (P) change in LCO with three oxygen vacancies created in the 40 -atom unit cell (see Fig. 1 in the main manuscript) as a function of the rotation angle $\alpha$. The pyramidal layer acquires a polarization, shown by the black arrows in (a), because of the pyramidal rotation $\left(\alpha=22.83^{\circ}\right)$; by gradually decreasing $\alpha$ to $0^{\circ}(\mathrm{b})$, the polarization in this layer vanishes.

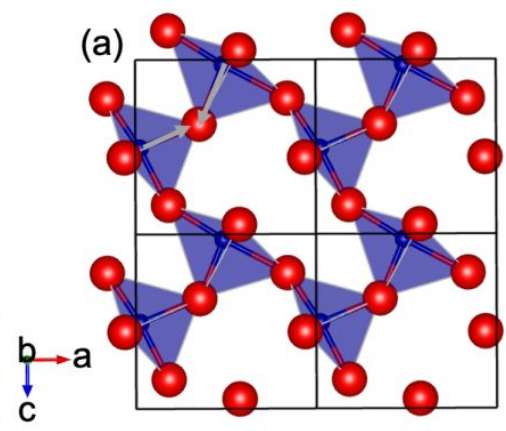

(b)

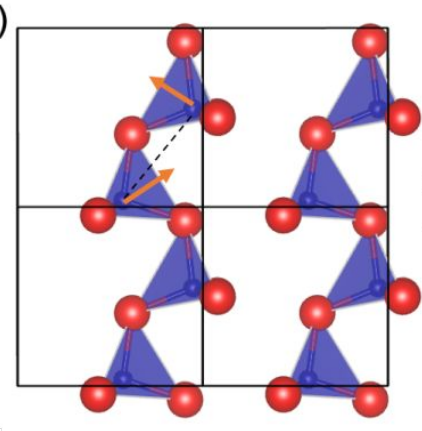

(c)

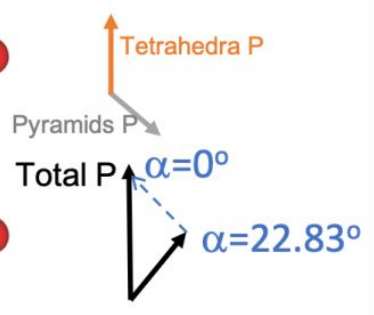

Figure S5 Polarization of pyramidal layer (a) and of tetrahedral layer (b) in the ground-state threevacancy structure. (c) The change of total polarization in the structure with pyramidal rotation angle $\alpha$.

Note that the tetrahedral layer in the three-vacancy structure has a similar tetrahedral rotation angle $\left(30^{\circ}\right)$ as in the two-vacancy structure $\left(31.5^{\circ}\right)$, thus we assume this layer has the same polarization value as in the two-vacancy case. However, the main polarization component in the three-vacancy case is now along the $c$ axis instead of the a axis, so in this case $P_{\text {perm_tetra }} \sim[0,0$, 7.7] $\left(\mu \mathrm{C} \mathrm{cm}{ }^{-2}\right)$.

When pyramids rotate from $\alpha=0^{\circ}$ to their equilibrium position $\left(22.83^{\circ}\right)$, the polarization of the whole structure along the a axis $\left(P_{\mathrm{a}}\right)$ increases, while it decreases along the $\mathrm{c}$ axis $\left(P_{\mathrm{c}}\right)$ because the polarizations of the pyramidal and tetrahedral layers partially cancel each other, as shown in Fig. S5c. The as-obtained polarization values from Berry phase calculations are shown in Table $\mathrm{S} 2$, from which we deduce that the value of $\mathbf{P}_{\text {Perm }}$ for the three-vacancy structure is $[3.1,0,5.0]$ $\left(\mu \mathrm{C} \mathrm{cm}^{-2}\right)$.

It worth mentioning that in the three-vacancy structure the polarization change with $\alpha$ is not linear, as we find a large increase of $P_{\mathrm{a}}$ from $\alpha=0^{\circ}$ to $11^{\circ}$, but only a slight decrease for larger angles, similar for $P_{\mathrm{c}}$. 
Table S2 Calculated polarization values $\left(\mu \mathrm{C} \mathrm{cm}^{-2}\right.$ ) for different pyramidal rotation angle $\alpha$ (see Fig. S4). When computing polarization along the $c$ axis for $\alpha=0^{\circ}$, we obtained results (11.2) for a different branch and the value $\frac{R e}{\Omega}\left(17.8 \mu \mathrm{C} \mathrm{cm}^{-2}\right)$ was subtracted in order to remain in the same branch. $\Delta P$ is the polarization change relative to the value obtained at $\alpha=0^{\circ}$ case, and its positive/negative sign represents the increasing/decreasing of $P_{\text {perm }}$ based on Fig. S5c.

\begin{tabular}{|l|l|l|l|l|}
\hline \multicolumn{1}{r|}{$\alpha$} & $0^{\circ}$ & $11^{\circ}$ & $15.2^{\circ}$ & $22.83^{\circ}$ \\
\hline$P_{\mathrm{a}}$ & & & & \\
\hline$\Delta P$ & 0.3 & 3.9 & 3.8 & 3.4 \\
\hline$P_{\mathrm{c}}$ & 0 & 3.6 & 3.5 & 3.1 \\
\hline$\Delta P$ & -6.6 & -6.8 & -3.2 & -3.9 \\
\hline & 0 & -0.2 & -3.4 & -2.7 \\
\hline
\end{tabular}

3. Calculation of vibrational free energy

Table S2 Computed vibrational free energy of the pristine perovskite LCO. We report in the table the unit cell (second row), the computational methods used (third row), the plane wave cutoff (fourth row) and k-point sampling resolution (fifth row).

\begin{tabular}{|l|l|l|l|l|}
\hline $\begin{array}{l}\text { Vibrational free } \\
\text { energy T=300 K } \\
\text { (ev/f.u.) }\end{array}$ & $0.116^{*}$ & $0.126^{*}$ & $0.133^{+}$ & $0.131^{\dagger}$ \\
\hline Structure & $\begin{array}{l}\text { 40-atom } \\
\text { orthorhombic } \\
\text { cell }\end{array}$ & $\begin{array}{l}\text { 10-atom } \\
\text { rhombohedral cell }\end{array}$ & $\begin{array}{l}\text { 10-atom } \\
\text { rhombohedral cell }\end{array}$ & $\begin{array}{l}\text { 20-atom } \\
\text { monoclinic cell }\end{array}$ \\
\hline Method & $\begin{array}{l}\text { DFPT+U } \\
(\Gamma \text { point })\end{array}$ & $\begin{array}{l}\text { DFPT+U } \\
(\Gamma \text { point })\end{array}$ & $\begin{array}{l}\text { Frozen phonon } \\
(100 \times 100 \times 100 \mathrm{q} \\
\text { points })\end{array}$ & $\begin{array}{l}\text { Frozen phonon } \\
\text { (100x100x100 } \\
\text { q points) }\end{array}$ \\
\hline Ecutwf $($ Ry) & 50 & 100 & 100 & 100 \\
\hline $\begin{array}{l}\text { K point } \\
\text { resolution }\left(\AA^{-1}\right)\end{array}$ & $0.03-0.05$ & 0.025 & 0.025 & 0.025 \\
\hline
\end{tabular}

* value obtained using Equation $17 ;{ }^{\dagger}$ value obtained directly from Phonopy.

We compared DFPT+U results for vibrational free energies computed with a 10-atom rhombohedral unit cell (see Fig. S6 for different unit cells used), and with different cutoffs (up to $100 \mathrm{Ry}$ ) and k-point resolution (up to $0.025 \AA^{-1}$ ). We then carried out calculations using the frozen phonon method as implemented in the Phonopy code with a dense q-point sampling (see Table S3. Calculations carried out with the Frozen phonon method using the 20 -atom monoclinic cell 
provided the most accurate results and are used here as our reference values. The DFPT+U method with 50 Ry plane-wave cutoff thus underestimates the vibrational free energy by 0.013 $\mathrm{eV} / \mathrm{f}$.u. (formula unit) in the perovskite phase. Such difference contributes to a $15 \%$ change in the vibrational energy, but only to a $\sim 2 \%$ change in $F_{\mathrm{vac} 0}$, therefore we considered the results obtained with the DFPT+U method and 50 Ry plane-wave cutoff as sufficiently accurate for the present study. We also note that the k-point resolution and plane-wave cutoff energy contributed the most to the underestimate, followed by q-point density, and finally the unit cell size (in the frozen phonon method).

(a)

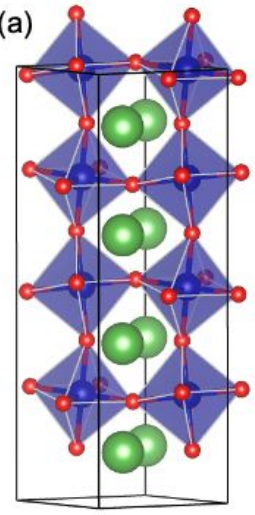

(b)

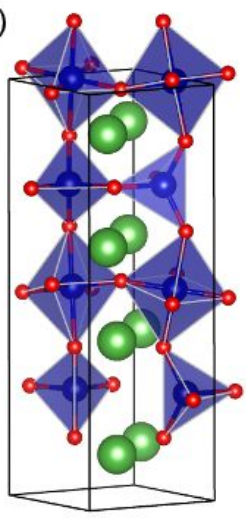

(c)

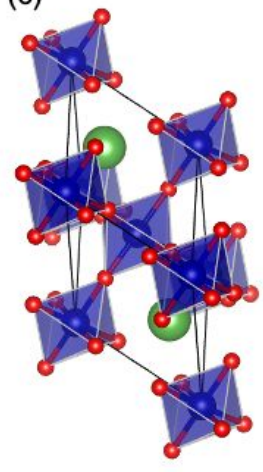

(d)

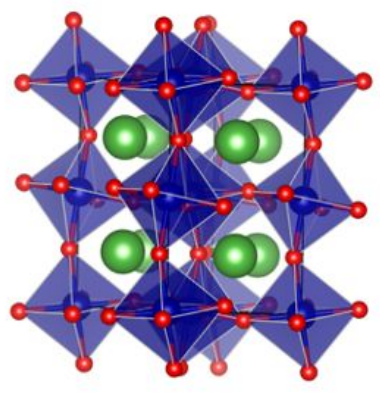

Figure $\mathbf{6 6}$ Cell structures used in phonon calculations. a) Perovskite phase, 40 -atom orthorhombic unit cell. b) BM phase, 40-atom orthorhombic unit cell. c) Perovskite phase in 10-atom rhombohedral unit cell. d) Perovskite phase, 20-atom monoclinic unit cell.

\section{Electric field range}

We briefly report the electric field values corresponding to the computed voltages, which can be obtained by dividing the voltage by the unit cell length in the considered direction. The obtained electric field varies between $0.5-1\left(\mathrm{GV} \mathrm{m}^{-1}=10^{9} \mathrm{~V} \mathrm{~m}^{-1}\right)$ along the $\mathrm{a}$ and $\mathrm{c}$ axes and varies between 1.2-3 ( $\left.\mathrm{GV} \mathrm{m}^{-1}\right)$ along the $b$ axis as a function of oxygen vacancy concentration. We note here in experimental films $\sim 50 \mathrm{~nm}$ thick or more, the electric field will be much smaller than the one calculated for the $\sim 1.5 \mathrm{~nm}$ case in our supercell. So, it's unlikely the electric field will reach the breakdown strength and make the material metallic before the transition occurs. We also note here the electric field is not considered as a means to generate oxygen vacancies, and is unlikely be able to do $\mathrm{so}^{8}$. Nevertheless, the electronic polarization may help decrease the oxygen vacancy formation energy, as shown in a previous study? 
References

(1) Floris, A.; de Gironcoli, S.; Gross, E. K. U.; Cococcioni, M. Vibrational Properties of MnO and $\mathrm{NiO}$ from DFT $+U$-Based Density Functional Perturbation Theory. Phys. Rev. B 2011, 84 (16), 161102. https://doi.org/10.1103/PhysRevB.84.161102.

(2) Floris, A.; Timrov, I.; Himmetoglu, B.; Marzari, N.; de Gironcoli, S.; Cococcioni, M. HubbardCorrected Density Functional Perturbation Theory with Ultrasoft Pseudopotentials. Phys. Rev. B 2020, 101 (6), 064305. https://doi.org/10.1103/PhysRevB.101.064305.

(3) Zak, J. Berry's Phase for Energy Bands in Solids. Phys. Rev. Lett. 1989, 62 (23), 2747-2750. https://doi.org/10.1103/PhysRevLett.62.2747.

(4) King-Smith, R. D.; Vanderbilt, D. Theory of Polarization of Crystalline Solids. Phys. Rev. B 1993, 47 (3), 1651-1654. https://doi.org/10.1103/PhysRevB.47.1651.

(5) Tajima, S.; Masaki, A.; Uchida, S.; Matsuura, T.; Fueki, K.; Sugai, S. Infrared Reflectivity and Electronic States in Perovskite-Type Oxides $\mathrm{La}_{1-x} \mathrm{Sr}_{\mathrm{x}} \mathrm{FeO}_{3}$ and $\mathrm{La}_{1-\mathrm{x}} \mathrm{Sr}_{\mathrm{x}} \mathrm{CoO}_{3}$. J. Phys. C: Solid State Phys. 1987, 20 (23), 3469-3484. https://doi.org/10.1088/0022-3719/20/23/016.

(6) Nomerovannaya, L. V.; Makhnev, A. A.; Streltsov, S. V.; Nekrasov, I. A.; Korotin, M. A.; Shiryaev, S. V.; Bychkov, G. L.; Barilo, S. N.; Anisimov, V. I. The Influence of the $\mathrm{Co}^{3+} \mathrm{Spin}$ State on the Optical Properties of $\mathrm{LaCoO}_{3}$ and $\mathrm{HoCoO}_{3}$. Journal of Physics: Condensed Matter 2004, 16 (28), 5129-5136. https://doi.org/10.1088/0953-8984/16/28/030.

(7) Jia, T.; Zeng, Z.; Q. Lin, H.; Duan, Y.; Ohodnicki, P. First-Principles Study on the Electronic, Optical and Thermodynamic Properties of $\mathrm{ABO}_{3}(\mathrm{~A}=\mathrm{La}, \mathrm{Sr}, \mathrm{B}=\mathrm{Fe}, \mathrm{Co})$ Perovskites. RSC Adv. 2017, 7 (62), 38798-38804. https://doi.org/10.1039/C7RA06542F.

(8) Schie, M.; Menzel, S.; Robertson, J.; Waser, R.; De Souza, R. A. Field-Enhanced Route to Generating Anti-Frenkel Pairs in HfO 2. Phys. Rev. Mater. 2018, 2 (3), 035002. https://doi.org/10.1103/PhysRevMaterials.2.035002.

(9) Youssef, M.; Van Vliet, K. J.; Yildiz, B. Polarizing Oxygen Vacancies in Insulating Metal Oxides under a High Electric Field. Phys. Rev. Lett. 2017, 119 (12), 126002. https://doi.org/10.1103/PhysRevLett.119.126002. 